Journal of Russian Laser Research, Volume 27, Number 4, 2006

Dedicated to the memory

of Andrey Vinogradov.

\title{
ENTANGLEMENT AND ITS OPERATIONAL MEASURE
}

\author{
Alexander A. Klyachko, Barış Öztop, and Alexander S. Shumovsky \\ Faculty of Science, Bilkent University, Bilkent, Ankara, 06800 Turkey \\ e-mail: shumo@fen.bilkent.edu.tr
}

\begin{abstract}
An operational representation of concurrence measuring the entanglement of bipartite systems by means of averages of basic observables is discussed. We prove the validity of this representation for bipartite systems with any dimension of a single-party Hilbert space. We show that Wigner-Yanase "skew" information gives a reasonable estimation of the amount of entanglement (in ebits) carried by mixed two-qubit states.
\end{abstract}

Keywords: entanglement, symmetry, uncertainty.

\section{Introduction}

In recent years, much effort has been devoted to the quantification of entanglement, which nowadays is considered to be one of the most fundamental features of quantum mechanics and a key physical resource of quantum information processing and quantum computing. A number of important results have been obtained in the field.

Namely, it has been shown that any measure of entanglement should be expressed in terms of the entanglement monotone [1]. This means that any measure of entanglement $\mu(\psi)$ represented by a functional of quantum states $\psi$ of a system defined in the Hilbert space $\mathbb{H}$ should decrease monotonically under the action of stochastic local operations assisted by classical communications (SLOCC) [2] on the state

$$
\mu([\widehat{\mathrm{SLCC}}] \psi) \leq \mu(\psi)
$$

In general, SLOCC are represented by operators from the complexified dynamic symmetry group $G^{c}[3,4]$

$$
[\widehat{\mathrm{SLOCC}}]=g^{c} \in G^{c} \text {. }
$$

In turn, the dynamic symmetry group $G$ is associated with the Lie algebra $\mathcal{L}$ of observables, whose measurement specifies the states $\psi \in \mathbb{H}[5-7]$. Note that SLOCC is able neither to create nor to destroy the entanglement $[2]$.

Manuscript submitted by the authors in English on April 12, 2006.

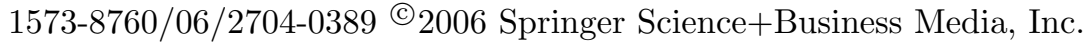


Using another language, one can say that a proper measure of the entanglement should be expressed in terms of geometrical invariants [5] peculiar to the system under consideration. In other words, the entanglement exists for states $\psi \in \mathbb{H}$ that can be separated from zero by a $G^{c}$-invariant function

$$
I\left(g^{c} \psi\right)=I(\psi) \neq 0 .
$$

Then, the measure of entanglement can be associated with the minimum length of a vector in the complex orbit [5]

$$
\mu(\psi)=\min _{g^{c} \in G^{c}}\left|g^{c} \psi\right|^{2} .
$$

(For a discussion of geometric invariants and their application in physics, see [8].)

For example, the concurrence [9] (measure of bipartite entanglement) and 3-tangle [10] (measure of entanglement carried by a pure three-qubit state) are expressed in terms of only geometrical invariants for the respective systems [11].

The creation of sources of entangled states for practical use requires a test of the amount of entanglement carried by the states. This implies the representation of the measure of entanglement in terms of physical quantities that can be directly measured. Note that conventional representations of concurrence $[9,12,13]$ are expressed in terms of complex coefficients specifying bipartite states. The direct measurement of those coefficients represents a quite sophisticated problem and most probably cannot be successfully realized at all.

Therefore, proposals to measure the entanglement via a specific correlation of local measurements [14-16] seem to be of high interest. In fact, such a representation directly follows the Bell's idea [17] that the quantum entanglement manifests itself by measuring the local observables.

The main goal of this paper is to discuss in detail a proposal that has been made in [16], according to which the concurrence of a pure two-qubit state can be represented in terms of averages of certain basic observables. We prove that this representation is valid for pure states of bipartite systems of any arbitrary dimension. We also discuss an extension of the approach to the case of mixed bipartite states and show that the so-called Wigner-Yanase skew information (WYSI) [18] gives a reasonable estimation of the amount of entanglement in this case.

This paper is arranged as follows.

In Sec. 2 we briefly discuss the relation between the dynamic symmetry and the choice of basic observables and, on the other hand, between the quantum uncertainties of those observables and entanglement. In Sec. 3 we compare different representations of the concurrence, including the operational representation of [16]. In Sec. 4 we prove that the operational representation is valid for pure states of bipartite systems of any arbitrary dimension. In Sec. 5 we discuss the application of the operational form of concurrence to mixed two-qubit states and show that WYSI gives a reasonable estimation of the entanglement carried by these states. Finally, in Sec. 6 we briefly discuss the results obtained and their further implementation.

\section{Basic Observables, Their Quantum Uncertainties, and Complete Entanglement}

Within the framework of conventional von Neumann theory of quantum measurements [19], all physical observables are assumed to be equally accessible. The development of quantum mechanics has shown 
that this is not true, at least for some special systems (see, e.g., [20,21]).

For example, in the case of a system of $N$ identical particles, each defined in the Hilbert space $\mathbb{H}$ of its internal degrees of freedom, the total space of states is reduced to the symmetric tensor $S^{N} \mathbb{H} \subset \mathbb{H}^{\otimes N}$ for bosons and to the skew symmetric tensors $\wedge^{N} \mathbb{H} \subset \mathbb{H}^{\otimes N}$ for fermions. This superselection rule imposes severe restrictions on the manipulation, with quantum states effectively reducing the accessible measurements to that of a single particle.

The quantum entanglement also falls off the framework of the von Neumann theory of measurements. For example, in the case of a bipartite system defined in the Hilbert space $\mathbb{H}=\mathbb{H}^{(A)} \otimes \mathbb{H}^{(B)}$, the von Neumann theory allows measurements corresponding to the total symmetry group SU(HI), while physically the entanglement is caused by a certain symmetry break provided by the reduction

$$
\mathrm{SU}\left(\mathbb{H}_{A}\right) \times \mathrm{SU}\left(\mathbb{H}_{B}\right) \subset \mathrm{SU}\left(\mathbb{H}_{A} \otimes \mathbb{H}_{B}\right)
$$

This makes essential only local measurements.

Thus, the specification of a quantum system should include the choice of proper measurements forming a representation of a certain Lie algebra $\mathcal{L}$ [20]. The dynamic symmetry of the system is then defined by the corresponding Lie group

$$
G=\exp (i \mathcal{L})
$$

Following $[4,5]$, we define basic observables $\left\{X_{i}\right\}$ peculiar to a given quantum system as the orthogonal basis of the corresponding Lie algebra $\mathcal{L}$.

For example, a qubit (state of spin-1/2 particle) can be characterized by the measurement of three spin operators (Pauli matrices) forming a basis of $\mathcal{L}=\mathrm{su}(2)$. This implies the dynamic symmetry $G=\mathrm{SU}(2)$. In turn, a system of $N$ qubits is specified by the symmetry

$$
G=\underbrace{\mathrm{SU}(2) \times \mathrm{SU}(2) \times \cdots \times \mathrm{SU}(2)}_{N \text { times }},
$$

and the total set of basic observables consists of $2 N$ (local) spin operators.

A qutrit (state of a quantum system defined in the three-dimensional Hilbert space) has general symmetry given by the $G=\mathrm{SU}(3)$ group, so that the measurements are provided by the eight independent Hermitian generators of the su(3) algebra $[22,23]$. The entanglement of qutrits is widely discussed in connection with the realization of ternary quantum logic [24].

At the same time, the reduction of symmetry to $\mathrm{SU}(2) \subset \mathrm{SU}(3)$ cuts the number of basic observables down to three spin-1 operators [25]. Such a SU(2) qutrit can be entangled as a single particle [25], while the entanglement of $\mathrm{SU}(3)$ qutrits is allowed beginning with bipartite systems.

This relativity of entanglement with respect to the choice of basic observables emphasizes the importance of analysis of dynamic symmetry in the description of entanglement.

According to the central limit theorem of the probability theory, even if we know moments of all orders, we cannot, in general, determine the probability distribution (wave function, in the quantum case). Therefore, only the averages $\left\langle\psi\left|X_{i}\right| \psi\right\rangle$ and variances (uncertainties)

$$
V_{i}(\psi)=\left\langle\psi\left|X_{i}^{2}\right| \psi\right\rangle-\left\langle\psi\left|X_{i}\right| \psi\right\rangle^{2}
$$

are usually discussed in the context of quantum measurements. The average gives the center of the distribution, while the uncertainty gives its semi-width. 
It has been noted in [26] that completely entangled states $\psi_{C E}$ obey the following condition:

$$
\forall i \quad\left\langle\psi_{\mathrm{CE}}\left|X_{i}\right| \psi_{\mathrm{CE}}\right\rangle=0
$$

where $X_{i}$ denote the basic observables and the subscript $i$ is set for both parties and single-party basic observables. According to $[4,7,26]$, we use Eq. (2) as an operational definition of the complete entanglement. Let us stress that the definition of complete entanglement (2) assumes the measurement of all basic observables in a given state.

Let us also stress that despite the long history of investigation of the entanglement, so far there is no agreement on the definition of this physical phenomenon. It is usually associated either with the violation of Bell's conditions [27] or with nonseparability of entangled states in the Hilbert space of a composite system [28]. Both definitions assume nonlocality of the entanglement.

Unfortunately, both definitions have quite restricted applicability. The point is that nonseparable states can be unentangled but, at the same time, they manifest the violation of Bell's conditions. An example of some considerable interest is provided by the so-called $W$-state of three qubits [2], which is unentangled [11] but shows the violation of Bell's conditions [29].

The point is that originally Bell's conditions were established to prove the absence of hidden classical variables in quantum mechanics [17]. This is a general feature of quantum mechanics, which is not peculiar to the entangled state alone. For detailed discussion, see $[5,30]$.

In turn, the notion of nonseparability (as well as nonlocality) has no meaning in the case of a single particle, which can also be entangled with respect to its intrinsic degrees of freedom $[7,25]$.

At the same time, condition (2) defines the complete entanglement irrespective of whether or not the system under consideration consists of a few separable parts.

As soon as completely entangled states $\psi_{\mathrm{CE}}$ are specified, all other entangled states $\psi_{\mathrm{E}}$ can be obtained from them by means of SLOCC

$$
\left|\psi_{\mathrm{E}}\right\rangle=[\widehat{S[O C} C]\left|\psi_{\mathrm{CE}}\right\rangle \equiv g^{c}\left|\psi_{\mathrm{CE}}\right\rangle, \quad g^{c} \in G^{c} .
$$

Through the use of definition (2), one can obtain infinitely many completely entangled states $\psi_{\mathrm{CE}} \in \mathbb{H}$ of the same system. Among them, special attention should be paid to generic entangled states, i.e., to completely entangled states with a simple structure (like EPR and GHZ states of two and three qubits, respectively), which can be used to construct an orthonormal basis of completely entangled states in $\mathbb{H}$ (see [31]).

In the case of basic observables $\left\{X_{i}\right\}$ associated with the orthogonal basis of a compact Lie algebra $\mathcal{L}$, the condition of complete entanglement (2) has an interesting interpretation. In view of the definition of quantum uncertainty (1), the condition (2) leads to the following [4,7]:

$$
\forall \psi_{\mathrm{CE}} \in \mathbb{H} \quad \mathbb{V}\left(\psi_{\mathrm{CE}}\right) \equiv \sum_{i} V_{i}\left(\psi_{\mathrm{CE}}\right)=\max _{\psi \in \mathbb{H}} \mathbb{V}(\psi)=C_{\mathbb{H}},
$$

where $\mathbb{V}(\psi)$ denotes the total uncertainty of basic observables in the state $\psi$ and $C_{\mathbb{H}}$ is the Casimir number

$$
\sum_{i} X_{i}^{2}=C_{\mathbb{H}} \times \mathbf{1}
$$

Here 1 denotes the identity operator. 
In other words, completely entangled states manifest the maximum amount of quantum uncertainties. Note that qudits [states of systems with dynamic symmetry group $\mathrm{SU}(n)$ ] always contain the Casimir operator (5) in the enveloping algebra of the compact Lie algebra of observables $\mathcal{L}=\operatorname{su}(n)$.

In fact, Eq. (4) can be considered as a variational principle similar, in a sense, to the maximumentropy principle used in statistical physics to define the thermal-equilibrium state [32]. This analogy may be deeper than it seems to be at first sight. Namely, Wigner and Yanase [18] claimed that uncertainty (1) specifies the amount of specific quantum information on the state $\psi \in \mathbb{H}$ that can be extracted from a macroscopic measurement of quantum observable $X_{i}$ in this state (for further discussion, see [33]). Leaving out a discussion of the exact information meaning of quantum uncertainty, we can conclude that conditions (2) and (4) define completely entangled states as those carrying the maximum amount of WYSI extracted from the measurement of all basic observables.

\section{Representations of Concurrence}

For clarity, let us begin with the discussion of the simplest two-qubit system.

This system is defined in the four-dimensional Hilbert space

$$
\mathbb{H}=\mathbb{H}^{(A)} \otimes \mathbb{H}^{(B)}, \quad \operatorname{dim} \mathbb{H}^{(A, B)}=2,
$$

with the dynamic symmetry $G=\mathrm{SU}(2) \times \mathrm{SU}(2)$ and observables given by the three Hermitian spin- $1 / 2$ operators (or Pauli matrices) that for each party can be presented as follows:

$$
\sigma_{x}=\left(\begin{array}{cc}
0 & 1 \\
1 & 0
\end{array}\right), \quad \sigma_{y}=\left(\begin{array}{rr}
0 & -i \\
i & 0
\end{array}\right), \quad \sigma_{z}=\left(\begin{array}{rr}
1 & 0 \\
0 & -1
\end{array}\right) .
$$

Since $\sigma_{\alpha}^{2}=\mathbf{1}$ for both parties and all $\alpha=x, y, z$, it is seen that the maximum amount of total uncertainty (4) for two qubits is $\mathbb{V}_{\max }=C_{\mathbb{H}}=6$. In the case of pure two-qubit state of the form

$$
|\psi\rangle=\sum_{\ell, \ell^{\prime}=0,1} \psi_{\ell \ell^{\prime}}|\ell\rangle \otimes\left|\ell^{\prime}\right\rangle, \quad \sum_{\ell, \ell^{\prime}=0}^{1}\left|\psi_{\ell \ell^{\prime}}\right|^{2}=1,
$$

the minimum value of total uncertainty $\mathbb{V}_{\min }=4$ is provided by unentangled (separable) states, e.g., by $|\ell\rangle \otimes|\ell\rangle$.

It is known that the only true measure of the entanglement of two qubits is provided by the concurrence [9], which is defined as follows:

$$
C(\rho)=\max \left\{0, \lambda_{1}-\lambda_{2}-\lambda_{3}-\lambda_{4}\right\} .
$$

Here $\rho$ is the density matrix of the systems and $\lambda_{i}$ 's $\left(\lambda_{1}=\max \left\{\lambda_{i}\right\}\right)$ denote square roots of the eigenvalues of non-Hermitian matrix

$$
\rho \tilde{\rho} \equiv \rho\left(\sigma_{y}^{(A)} \sigma_{y}^{(B)} \rho^{*} \sigma_{y}^{(A)} \sigma_{y}^{(B)}\right)
$$

where

$$
\sigma_{j}^{(A)}=\sigma_{j} \otimes 1, \quad \sigma_{j}^{(B)}=\mathbf{1} \otimes \sigma_{j}
$$


denote the $j$ th Pauli operator $(j=x, y, z)$ defined for either party in the two-qubit Hilbert space.

The above used wording "the only true measure" means that all true measures of two-qubit entanglement should be expressed in terms of the functions of concurrence (8).

In the case of a pure normalized two-qubit state (7), the expression for concurrence (8) is reduced to

$$
C(\psi)=2|\operatorname{det}[\psi]|
$$

where $[\psi]$ is a $2 \times 2$ matrix of complex coefficients on the right-hand side of Eq. (7). Note that (9) represents the only geometric invariant for two qubits.

It was shown in [12] that concurrence (8) for pure state (7) can also be represented in the following way:

$$
C(\psi)=\sqrt{2\left[1-\operatorname{Tr}\left(\rho_{r}^{2}\right)\right]}
$$

where $\rho_{r}$ denotes the reduced (single-party) density matrix

$$
\rho_{r}=\operatorname{Tr}^{(A)} \rho=\operatorname{Tr}^{(B)} \rho, \quad \rho=|\psi\rangle\langle\psi| .
$$

Unlike the original representation of pure-state concurrence, Eq. (10) gives a measure of the entanglement for bipartite systems of any arbitrary dimension $n=\operatorname{dim} \mathbb{H}^{(A, B)}[12]$ (see [13] for further discussion). To keep the value of concurrence between 0 for unentangled and 1 for completely entangled states, the factor 2 in the square root in Eq. (10) should be changed to $\nu=n /(n-1)$.

Both representations (9) and (10) express the concurrence in terms of complex coefficients specifying the state (7). The measurement of those coefficients does not seem to be an easy problem. In fact, taking into account the normalization conditions in $(7)$, we can conclude that the determinant $\operatorname{det}[\psi]$ in Eq. (9) is specified by seven real parameters, while the number of observables [Pauli operators (6) for both parties] is equal to 6 .

Following [16], consider now another representation of concurrence (9) that can be directly expressed in terms of the total uncertainty $\mathbb{V}(\psi)$. Calculating the averages of basic observables (6) for both parties in the state (7), for the total uncertainty we obtain

$$
V(\psi)=4+4\left(\left|\psi_{00}\right|^{2}\left|\psi_{11}\right|^{2}+\left|\psi_{01}\right|^{2}\left|\psi_{10}\right|^{2}\right)-8\left|\psi_{00}\right|\left|\psi_{11}\right|\left|\psi_{01}\right|\left|\psi_{10}\right| \cos \phi
$$

where

$$
\phi=\arg \psi_{00}+\arg \psi_{11}-\arg \psi_{01}-\arg \psi_{10}
$$

It is now a straightforward matter to show that the concurrence (9) can be expressed in terms of the total variance (11) as follows:

$$
C(\psi)=\sqrt{\frac{\mathbb{V}(\psi)-4}{2}} .
$$

Taking into account that $\mathbb{V}_{\text {min }}=4$ and $\mathbb{V}_{\max }-\mathbb{V}_{\min }=2$ for two qubits, we can rewrite this equation in the following way:

$$
C_{\mathrm{op}}(\psi)=\sqrt{\tilde{\mathbb{V}}(\psi)}, \quad \tilde{\mathbb{V}}(\psi)=\frac{\mathbb{V}(\psi)-\mathbb{V}_{\min }}{\mathbb{V}_{\max }-\mathbb{V}_{\min }}
$$


Hereafter we call Eq. (12) the operational representation of concurrence. In view of Eq. (5), we have

$$
\mathbb{V}(\psi)=\mathbb{V}_{\max }-R(\psi), \quad R(\psi)=\sum_{\alpha, j}\left\langle\psi\left|\sigma_{\alpha}^{(j)}\right| \psi\right\rangle^{2},
$$

so that the normalized total variance in Eq. (14) takes the form

$$
\tilde{\mathbb{V}}(\psi)=1-\frac{R(\psi)}{R_{\min }}
$$

where $R_{\min }=\min R(\psi)$ denotes the minimum value of quadratic form $R$ in Eq. (13) provided by unentangled (separable) state. For two qubits, $R_{\min }=2$.

Thus, the operational representation (12) expresses the concurrence of two qubits (9) directly in terms of averages of basic observables.

Representation (12) can also be used as a measure of entanglement in the case of a single $S U(2)$, i.e., the state of a spin-1 like object [25]. The basic observables (components of spin-1 operator) have the form

$$
S_{x}=\left(\begin{array}{ccc}
0 & 1 / \sqrt{2} & 0 \\
1 / \sqrt{2} & 0 & 1 / \sqrt{2} \\
0 & 1 / \sqrt{2} & 0
\end{array}\right), \quad S_{y}=\left(\begin{array}{ccc}
0 & -i / \sqrt{2} & 0 \\
i / \sqrt{2} & 0 & -i / \sqrt{2} \\
0 & i / \sqrt{2} & 0
\end{array}\right), \quad S_{z}=\left(\begin{array}{ccc}
1 & 0 & 0 \\
0 & 0 & 0 \\
0 & 0 & -1
\end{array}\right)
$$

defined in the basis of states $|s\rangle, s= \pm 1,0$ spanning the Hilbert space $\mathbb{H}_{1}$. It is seen that the maximum uncertainty given by the Casimir number (4) is $C_{\mathbb{H}}=2$ in this case, while the minimum value is $\mathbb{V}_{\min }=1$. Thus, the formal use of operational representation (12) gives

$$
C_{\mathrm{op}}(\psi)=\sqrt{\mathbb{V}(\psi)-1}
$$

for pure states of the form

$$
|\psi\rangle=\sum_{s=-1}^{1} \psi_{s}|s\rangle, \quad \sum_{s=-1}^{1}\left|\psi_{s}\right|^{2}=1
$$

It is a straightforward matter to express Eq. (17) in terms of coefficients of this states as follows:

$$
C(\psi)=2\left|\psi_{+1} \psi_{-1}-\frac{\psi_{0}^{2}}{2}\right|
$$

which resembles Eq. (9) for the concurrence in two-qubit state (7).

In a sense, spin-1 system is similar to two qubits defined in the symmetric subspace of the Hilbert space $\mathbb{H}_{1 / 2} \otimes \mathbb{H}_{1 / 2}$ of two spin- $1 / 2$ like objects. In fact, this can be seen in the Clebsch-Gordan decomposition

$$
\mathbb{H}_{\frac{1}{2}} \otimes \mathbb{H}_{\frac{1}{2}}=\mathbb{H}_{1} \oplus \mathbb{H}_{0}
$$

where $\mathbb{H}_{0}$ denotes a one-dimensional subspace generated by antisymmetric two-qubit state

$$
|A\rangle=\frac{1}{2}(|0,1\rangle-|1,0\rangle)
$$


At the same time, there is the following correspondence between the symmetric states of two qubits and states $|s\rangle$ of a single $S U(2)$ qutrit:

$$
|0,0\rangle=|+1\rangle, \quad \frac{1}{2}(|0,1\rangle+|1,0\rangle)=|0\rangle, \quad|1,1\rangle=|-1\rangle .
$$

This allows us to reduce the two-qubit state (7) to a single $S U(2)$ qutrit state (17) in the Hilbert space with a discarded antisymmetric part. Equation (18) gives the concurrence (9) for such a reduced twoqubit system.

Let us stress that antisymmetric basis states (19) are not allowed for some physical systems. For example, photon twins form two qubits with respect to their polarization with only symmetric basis states (20) [34]. Hence, a biphoton [35] (photon twins created simultaneously and propagating in the same direction) represents the $S U(2)$ qutrit rather than $S U(3)$ qutrit. For more physical examples of $S U(2)$ qutrits, see [7,25].

In the next section, we prove that the operational representation of concurrence (12) can also be used as a measure of the entanglement for bipartite systems with any arbitrary dimension $n$ of a single-party Hilbert space.

\section{Bipartite System of Arbitrary Dimension}

It seems convenient to prove the validity of representation (12) in general settings without specification of parties of the system.

It is seen from Eqs. (13) and (14) that the operational representation of concurrence depends on the state $\psi$ via only the quadratic form $R(\psi)$, which in general settings can be presented as follows:

$$
R(\psi)=\sum_{i}\left[\operatorname{Tr}\left(\rho X_{i}\right)\right]^{2}, \quad \rho=|\psi\rangle\langle\psi| .
$$

Consider the space Herm $(\mathbb{H})$ of all Hermitian operators in $\mathbb{H}$ with the trace metric

$$
(X, Y)=\operatorname{Tr}_{\mathbb{H}}(X Y) .
$$

Let $\rho_{\mathcal{L}}$ be the orthogonal projection of $\rho \in \operatorname{Herm}(\mathbb{H})$ onto subalgebra $\mathcal{L} \subset \operatorname{Herm}(\mathbb{H})$, so that

$$
\operatorname{Tr}_{\mathbb{H}}(\rho X)=\operatorname{Tr}_{\mathbb{H}}\left(\rho_{\mathcal{L}} X\right) .
$$

We can now choose $\rho_{\mathcal{L}} / \sqrt{\left(\rho_{\mathcal{L}}, \rho_{\mathcal{L}}\right)} \in \mathcal{L}$ as one of the unit basis vectors $X_{i} \in \mathcal{L}$ (a basic observable). The remaining basis vectors are orthogonal to $\rho_{\mathcal{L}}$, so that

$$
\operatorname{Tr}\left(R_{\rho}\right)=\sum_{i}\left[\operatorname{Tr}\left(\rho_{\mathcal{L}} X_{i}\right)\right]^{2}=\frac{\operatorname{Tr}_{\mathbb{H}}\left(\rho_{\mathcal{L}}^{2}\right)^{2}}{\left(\rho_{\mathcal{L}}, \rho_{\mathcal{L}}\right)}
$$

The invariant-trace form which appears here is proportional to the Killing form of $\mathcal{L}$

$$
\operatorname{Tr}_{\mathbb{H}}(X Y)=D_{\mathbb{H}} \cdot(X, Y), \quad X, Y \in \mathcal{L},
$$

with the coefficient $D_{\mathbb{H}}$ known as the Dynkin index (concerning the Killing form and Dykin index, see [36]). Now, the total uncertainty can be written as follows:

$$
\mathbb{V}(\psi)=C_{\mathbb{H}}-D_{\mathbb{H}} \operatorname{Tr}_{\mathbb{H}}\left(\rho_{\mathcal{L}}^{2}\right),
$$


where $C_{\mathbb{H}}$ again denotes the Casimir number (4).

For the Lie algebra $\mathcal{L}$ under consideration, the coefficients $C_{\mathbb{H}}$ and $D_{\mathbb{H}}$ are given by the following equations:

$$
C_{\mathbb{H}}=(\lambda, \lambda+2 \delta), \quad D_{\mathbb{H}}=\frac{\operatorname{dim} \mathbb{H}}{\operatorname{dim} \mathcal{L}}(\lambda, \lambda+2 \delta),
$$

where $\lambda$ denotes the highest weight of irreducible representation $\mathbb{H}$ and $2 \delta$ is the sum of positive roots of $\mathcal{L}$.

In general, the Lie algebra $\mathcal{L}$ splits into a direct sum of simple components $\mathcal{L}=\bigoplus_{j} \mathcal{L}^{(j)}$, and each of its irreducible representation $\mathbb{H}$ decomposes into the tensor product $\mathbb{H}=\bigotimes_{j} \mathbb{H}^{(j)}$ of irreducible representations $\mathcal{L}^{(j)}: \mathbb{H}^{(j)}$. Under this setting, the Casimir number and Dynkin index can be found from the following additivity property:

$$
C_{\mathbb{H}}=\sum_{j} C_{\mathbb{H}}^{(j)}, \quad \frac{D_{\mathcal{L}}}{\operatorname{dim} \mathbb{H}}=\sum_{j} \frac{D_{\mathbb{H}^{(j)}}}{\operatorname{dim} \mathbb{H}^{(j)}} .
$$

In the case of the bipartite systems under consideration, index $j=A, B, \operatorname{dim} \mathbb{H}^{(A)}=\operatorname{dim} \mathbb{H}^{(B)}$, and the dynamic symmetry group

$$
G=\mathrm{SU}\left(\mathbb{H}^{(A)}\right) \times \mathrm{SU}\left(\mathbb{H}^{(B)}\right) .
$$

The corresponding Lie algebra $\mathcal{L}=\operatorname{Lie}(G)$ consists of the operators

$$
X=X^{(A)} \otimes \mathbf{1}+\mathbf{1} \otimes X^{(B)},
$$

where $X^{(A, B)}$ are the traceless Hermitian operators in $\mathbb{H}^{(A)}$ and $\mathbb{H}^{(B)}$. By the definition of reduced states $\rho^{(A)}, \rho^{(B)}$, we have

$$
\operatorname{Tr}_{\mathbb{H}}(\rho X)=\operatorname{Tr}_{\mathbb{H}}\left(\rho^{(A)} X^{(A)}\right)+\operatorname{Tr}_{\mathbb{H}}\left(\rho^{(B)} X^{(B)}\right)
$$

and, therefore, the projection $\rho_{\mathcal{L}}$ of $\rho$ into $\mathcal{L}$ characterized by the equation

$$
\operatorname{Tr}_{\mathbb{H}}(\rho X)=\operatorname{Tr}_{\mathbb{H}}\left(\rho_{\mathcal{L}} X\right)
$$

amounts to

$$
\rho_{\mathcal{L}}=\frac{1}{n}\left(\rho_{0}^{(A)} \otimes \mathbf{1}+\mathbf{1} \otimes \rho_{0}^{(B)}\right)
$$

where the subscript 0 denotes the traceless parts of $\rho^{(A, B)}$. A simple calculation shows that $\operatorname{Tr}\left(\rho_{\mathcal{L}}^{2}\right)$ is a linear function in $\operatorname{Tr}\left(\left[\rho^{(A)}\right]^{2}\right)$ and $\operatorname{Tr}\left(\left[\rho^{(B)}\right]^{2}\right)$. It is also seen that for the pure state under consideration

$$
\operatorname{Tr}\left(\left[\rho^{(A)}\right]^{2}\right)=\operatorname{Tr}\left(\left[\rho^{(B)}\right]^{2}\right) \equiv \operatorname{Tr}\left(\rho_{r}^{2}\right)
$$

and that, in this case, both the total uncertainty $\mathbb{V}(\psi)$ and the square of concurrence (10) [with factor $\nu=n /(n-1)$ instead of 2] are linear functions of $\operatorname{Tr}\left(\rho_{r}^{2}\right)$. Since the two linear functions $[C(\psi)]^{2}$ and normalized total uncertainty (14) have equal values for separable (unentangled) and completely entangled states, they are identical. 


\section{Entanglement of Mixed Bipartite States}

In the previous section, we have shown that Eq. (12) for the concurrence is valid for pure bipartite states of any dimension $n$. There is a problem of evaluation of the amount of entanglement carried by a bipartite mixed state. An exact measure is known only for the two-qubit case [concurrence (8)]. It seems to be interesting to check whether or not the operational representation of concurrence (12) fits for the measurement of mixed bipartite entangled states.

As an example of some considerable interest, we examine a one-parameter mixed two-qubit state specified by the density matrix

$$
\rho(x)=x \rho_{\text {chaotic }}+(1-x)\left|\psi^{(+)}\right\rangle\left\langle\psi^{(+)}\right|, \quad x \in[0,1]
$$

(the so-called Werner state [37]). Here $\rho_{\text {chaotic }}$ is a completely chaotic classical state with the density matrix

$$
\rho_{\text {chaotic }}=\frac{1}{4} \times \mathbf{1}=\left(\begin{array}{cccc}
1 / 4 & 0 & 0 & 0 \\
0 & 1 / 4 & 0 & 0 \\
0 & 0 & 1 / 4 & 0 \\
0 & 0 & 0 & 1 / 4
\end{array}\right)
$$

and

$$
\left|\psi^{(+)}\right\rangle=\frac{1}{\sqrt{2}}(|01\rangle+|10\rangle)
$$

is a completely entangled two-qubit state.

Thus, at $x=0$ the Werner state corresponds to a pure completely entangled state (23), while at $x=1$ it becomes a completely chaotic classical state (7). The state (21) is widely used for the investigation of separability and nonlocality of mixed states (see, e.g., [38] and references therein) and in the context of realization of quantum purification protocols [39].

In the case of Werner state (21), concurrence (8) takes the following form:

$$
C(\rho(x))=\left\{\begin{array}{ll}
1-3 x / 2, & 0 \leq x<2 / 3 \\
0, & 2 / 3 \leq x \leq 1
\end{array} .\right.
$$

We can also formally define the operational concurrence

$$
C_{\mathrm{op}}(\rho(x))=\sqrt{\frac{\mathbb{V}(\rho(x))-\mathbb{V}_{\min }}{\mathbb{V}_{\max }-\mathbb{V}_{\min }}} .
$$

By direct calculation, we get $\mathbb{V}(\rho(x))=\mathbb{V}_{\max }$ for all $x \in[0,1]$, so that the operational concurrence $(25)$ is always equal to 1 as if the Werner state (21) is a completely entangled one at any $x$. This undoubtedly wrong result comes from the fact that quantum and classical fluctuations caused by different terms in Eq. (21) contribute equally to the total uncertainty:

$$
\mathbb{V}(\rho(x))=x \mathbb{V}\left(\rho_{\text {chaotic }}\right)+(1-x) \mathbb{V}\left(\left|\psi^{(+)}\right\rangle\left\langle\psi^{(+)}\right|\right)=x \mathbb{V}_{\max }+(1-x) \mathbb{V}_{\max }=\mathbb{V}_{\max } .
$$


In other words, the total variance is not capable of distinguishing between quantum and classical uncertainties. The latter are caused by the statistical nature of mixed states.

Thus, the operational concurrence (12) based on the properties of total uncertainty cannot be used as a measure of the entanglement of mixed two-qubit states.

The original form that has been proposed in [18] to define the WYSI of the measurement of observable $X_{i}$ in a state $\rho$ is

$$
W_{i}(\rho)=-\frac{1}{2} \operatorname{Tr}\left(\left[X_{i}, \rho^{1 / 2}\right]^{2}\right)=\operatorname{Tr}\left(\rho X_{i}^{2}\right)-\operatorname{Tr}\left(\rho^{1 / 2} X_{i} \rho^{1 / 2} X_{i}\right) .
$$

In the case of a pure state with the density matrix $\rho=|\psi\rangle\langle\psi|$, it coincides with uncertainty (1).

In direct analogy to the total uncertainty, one can define the total WYSI by summation over all basic observables in Eq. (26):

$$
\mathbb{W}(\rho)=C_{\mathbb{H}}-\sum_{i} \operatorname{Tr}\left(\rho^{1 / 2} X_{i} \rho^{1 / 2} X_{i}\right)
$$

It is now seen that for the classical completely chaotic state (22), the second term on the right-hand side of Eq. (27) coincides with the first term up to the sign, so that $\mathbb{W}\left(\rho_{\text {chaotic }}\right)=0$ unlike the total uncertainty. Thus, the total WYSI seems to be a more realistic candidate to measure the entanglement carried by mixed states.

Since the WYSI is usually associated with a certain "information" of entropy [18,33], it seems natural to compare (27) directly with the entanglement of formation [9]

$$
\mathcal{E}(\rho)=h\left(\frac{1-\sqrt{1-C^{2}(\rho)}}{2}\right)
$$

rather than with the concurrence. The form (28) measures the amount of specific entanglement information (in ebits). Here $C(\rho)$ denotes the concurrence (8) and

$$
h(t) \equiv-t \log _{2} t-(1-t) \log _{2}(1-t) .
$$

Inasmuch as $\mathcal{E}$ ranges from zero (for unentangled states) to one (for completely entangled states), we compare it with the normalized total WYSI

$$
\tilde{\mathbb{W}}(\rho)=\frac{\mathbb{W}(\rho)-\mathbb{W}_{\min }}{\mathbb{W}_{\max }-\mathbb{W}_{\min }}
$$

In the case of Werner state (21), it takes the form

$$
\tilde{\mathbb{W}}(\rho(x))=\frac{1}{6} \mathbb{W}(\rho(x)) .
$$

The behavior of the normalized total WYSI (29) and the entanglement of formation (28) with the concurrence (24) as functions of $x$ is compared in Fig. 1. It is seen that the form (29) gives a reasonable estimation of the entanglement of formation, at least in the region with a considerable amount of entanglement $\mathcal{E}>0.1$ ebit. 


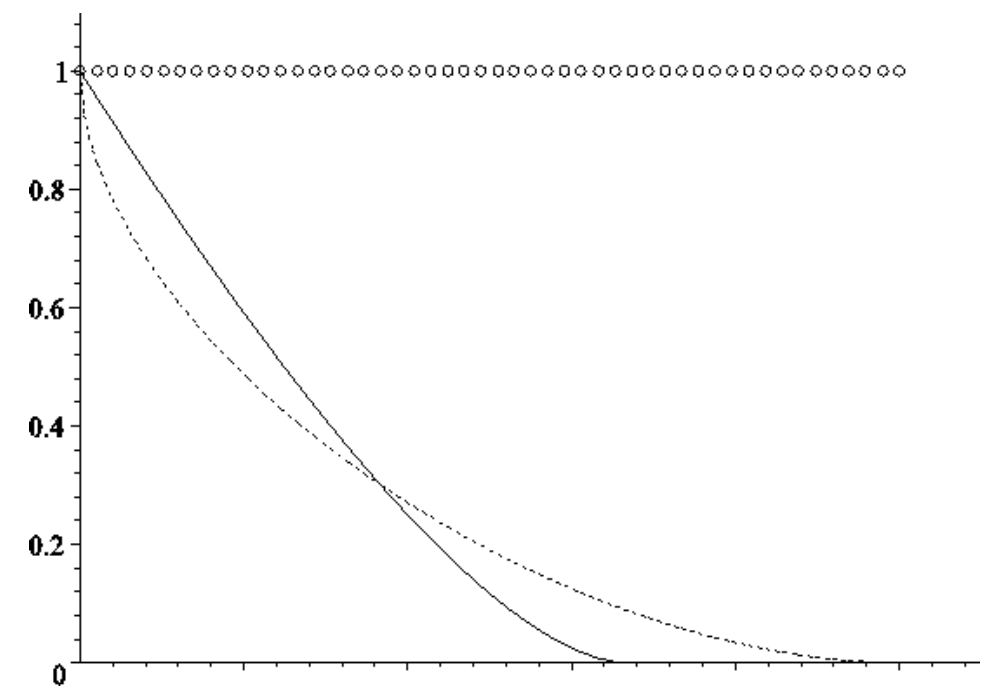

Fig. 1. Entanglement of formation (28) (solid line), normalized total WYSI (29) (dotted line), and operational concurrence (12) (circles) versus parameter $x$ for the Werner state (21).

\section{Conclusions}

We have reviewed the dynamic-symmetry-group approach to quantum entanglement which provides a unifying conceptual framework for the general description of entanglement as a physical phenomenon in an arbitrary physical system. The foundation stones of the approach are the choice of basic observables as the orthogonal basis of Lie algebra related to the dynamic-symmetry group of the physical system, the definition of completely entangled states by means of the extremal behavior of quantum uncertainties of basic observables, and the generation of entangled states from completely entangled states through the use of SLOCC. Unlike the conventional definitions, our approach neither assumes the nonlocality of the system nor the nonseparability of the corresponding Hilbert space. It instead links the entanglement with a specific behavior of quantum uncertainties of basic observables, which reveals the physical nature of this phenomenon.

We have shown that the concurrence for a bipartite pure state with any arbitrary dimension of singleparty Hilbert space can be presented in the form given by Eq. (14). Unlike the form (10), which deals with complex coefficients of bipartite state, Eq. (14) expresses the concurrence directly in terms of averages of basic observables. It should be emphasized that to specify whether or not a given state belongs to the class of entangled state, averages of all basic observables should be measured.

In addition to the case of pure states, we have shown that the Wigner-Yanase skew information (WYSI) can provide a reasonable estimation of the total amount of specific entanglement information carried by a mixed bipartite state. The form of the total WYSI (27) allows one to calculate only the quantum contribution to the uncertainty of all basic observables in a mixed state. More detailed investigation of the relation between the WYSI and entanglement information deserves a special consideration. The tomographic formulation of quantum mechanics may provide a convenient tool for this aim (see, e.g., [40] and references therein). 
Journal of Russian Laser Research

\section{Acknowledgments}

The authors express their gratitude to Dr. Margarita A. Man'ko, Dr. A. G. Molchanov, and Dr. J. Janzsky for excellent organization of the Workshop dedicated to the memory of the untimely death of A. V. Vinogradov, whose works have made a significant contribution to quantum optics. One of the authors (B.Ö.) thanks TUBITAK (Turkish Council for Science and Technology) for the support of the research project.

\section{References}

1. G. Vidal, J. Mod. Opt., 47, 355 (2000).

2. W. Dür, G. Vidal, and J.I. Cirac, Phys. Rev. A, 62, 06231 (2000).

3. F. Verstraete, J. Dehaene, and B. De Moor, Phys. Rev. A, 68, 012103 (2003).

4. A. A. Klyachko and A. S. Shumovsky, J. Opt. B: Quantum Semiclass. Opt., 5, S322 (2003).

5. A. A. Klyachko, E-print quant-ph/0206012.

6. A. A. Klyachko and A. S. Shumovsky, J. Opt. B: Quantum Semiclass. Opt., 6, S29 (2004).

7. A. A. Klyachko and A. S. Shumovsky, E-print quant-ph/0512213; J. Phys. Conf. Ser., 36, 87 (2006).

8. E. Vinberg and V. Popov, Invariant Theory, Springer, Berlin (1992).

9. S. Hill and W. K. Wootters, Phys. Rev. Lett., 78, 5022 (1997).

W.K. Wooters, Phys. Rev. Lett. 80, 2245 (1998).

10. V. Coffman, J. Kundu, and W. K. Wootters, Phys. Rev. A, 61, 052306 (2000).

11. A. Miyake, Phys. Rev. A, 67, 012108 (2003).

12. P. Rungta, V. Bužek, C. M. Caves, M. Hillery, and G. J. Milburn, Phys. Rev. A, 64, 042315 (2001).

13. F. Minnert, M. Kuś, and A. Buchleitner, Phys. Rev. Lett., 92, 167902 (2004).

14. F. Verstraete, M. A. Martin-Delgado, and J. I. Cirac, Phys. Rev. Lett. 92, 087201 (2004).

15. B.-Q. Jin and V. E. Korepin, Phys. Rev. A, 69, 062314 (2004).

16. A. A. Klyachko, B. Öztop, and A. S. Shumovsky, Appl. Phys. Lett., 88, 124102 (2006).

17. J. S. Bell, Rev. Mod. Phys., 38, 447 (1966).

18. E. P Wigner, Z. Phys., 131, 101 (1952).

E. P. Wigner and M. M. Yanase, Proc. Natl. Acad. Sci. USA, 19, 910 (1963).

19. J. von Neumann, Mathematical Foundations of Quantum Mechanics, Princeton University Press, Princeton (1996).

20. R. Hermann, Lie Groups for Physicists, Benjamin, New York (1966).

21. G. G. Emch, Mathematical and Conceptual Foundations of 20th Century Physics, North Holland, Amsterdam (1984).

22. C. M. Caves and G. J. Milburn, Opt. Commun., 179, 439 (2000).

23. A. T. Bölükbaşı and T. Dereli, E-print quant-ph/0511111; J. Phys. Conf. Ser., 36, 28 (2006).

24. H. Bechman-Pasquinucci and A. Peres, Phys. Rev. Lett., 85, 3313 (2000).

D. Bruss and C. Macchiavello, Phys. Rev. Lett., 88, 127901 (2002).

G. A. Maslennikov, A. A. Zhukov, M. V. Chekhova, and S. P. Kulik, J. Opt. B: Quantum Semicalss. Opt., 5, 530 (2003).

D. Kaszlikowski, D. K. L. Oi, M. Christandl, K. Chang, A. Ekert, L. C. Kwek, and C. H. Oh, Phys. Rev. A, 67, 012310 (2003). 
T. Durt T., N. Cerf, N. Gisin, and M. Zukowski, Phys. Rev. A, 67, 012311 (2003).

T. Durt, D. Kaszlikowski, J.-L. Chen, and L. C. Kwek, Phys. Rev. A, 69, 032313 (2004).

25. M. A. Can, A. A. Klyachko, and A. S. Shumovsky, J. Opt. B: Quantum Semiclass. Opt., 7, L1 (2005).

26. M. A. Can, A. A. Klyachko, and A. S. Shumovsky, Phys. Rev. A, 66, 02111 (2002).

27. M. Nielsen and I. Chuang, Quantum Computation and Quantum Information, Cambridge University Press, New York (2000).

28. D. Bruß, J. Math. Phys., 43, 4237 (2002).

29. A. Cabello, Phys. Rev. A, 65, 032108 (2002).

30. A. A. Klyachko, E-print quant-ph/0511102; J. Phys. Conf. Ser., 36, 72 (2006).

31. A. Binicioğlu, Ö. Çakır, A. A. Klyachko, and A. S. Shumovsky, Int. J. Quantum Inform., 3, 661 (2005).

32. L. D. Landau and E. M. Lifshitz, Statistical Physics, Pergamon Press, Oxford (1959).

33. H. Araki and M. M. Yanase, Phys. Rev., 120, 622 (1960).

F. J. Dyson and A. Leonard, J. Math. Phys., 8, 423 (1967).

F. J. Dyson, J. Math. Phys., 8, 1538 (1967).

E. H. Lieb and M. B. Ruskai, Phys. Rev. Lett., 30, 434 (1973).

A. Uhlman, Comm. Math. Phys., 54, 21 (1977).

M. Ozawa, Phys. Rev. Lett., 88, 050402 (2002).

J. Řeháček and Z. Hradil, Phys. Rev. Lett., 90127904 (2003).

S.-L. Luo, Phys. Rev. Lett., 91, 180403 (2003).

P. Gibilisco and T. Isola, J. Math. Phys., 44, 3752 (2003).

S.-L. Luo and Q. Zhang, Phys. Rev. A, 69, 032106 (2004).

34. V. B. Berestetskii, E. M. Lifshitz, and L. P. Pitaevskii, Quantum Electrodynamics, Pergamon Press, Oxford (1982).

35. A. V. Burlakov, M. V. Chekhova, O. A. Karabutova, D. N. Klyshko, and S. P. Kulik, Phys. Rev. A, 60, R4209 (1999).

M. B. Nasr, A. F. Abouraddy, M. C. Booth, B. E. A. Saleh, A. V. Sergienko, M. C. Teich, U. Kempe, and R. Wollescheensky, Phys. Rev. A, 65, 023816 (2002).

G. DiGiuseppe, M. Atature, M. Shaw, A. V. Sergienko, B. E. A. Saleh, M. C. Teich, A. J. Miller, S. W. Nam, and J. M. Martinis, Phys. Rev. A, 68, 063817 (2003).

Yu. I. Bogdanov, M. V. Chekhova, S. P. Kulik, G. A. Maslennikov, A. A. Zhukov, C. H. Oh, and M. K. Tey, Phys. Rev. Lett., 93, 230503 (2004).

36. A. L. Onishchik and E. B. Vinberg (eds.), Lie Groups and Lie Algebras III, Encyclopedia of Math. Science, Springer, Berlin (1994), Vol. 41.

37. R. F. Werner, Phys. Rev. A, 40, 4277 (1989).

38. T. Hiroshima and S. Ishizaka, Phys. Rev. A, 62, 044302 (2000).

39. M. Murano, M. B. Plenio, S. Popesku, V. Vedral, and P. L. Knight, Phys. Rev. A, 57, R4075, R4078 (1998).

40. V. A. Andreev and V. I. Man'ko, J. Opt. B: Quantum Semiclass. Opt., 2122 (2000).

O. V. Man'ko, V. I. Man'ko, G. Marmo, A. Shaji, E. C. G. Sudarshan, and F. Zaccaria, Phys. Lett. A, 339, 194 (2005). 